\title{
Topological weak mixing and diffusion at all times for a class of Hamiltonian systems
}

\author{
BASSAM FAYAD $\dagger$ and MARIA SAPRYKINA $\ddagger$ \\ $\dagger$ IMJ-PRG CNRS, UP7D, 58-56, avenue de France, Boite Courrier 7012, \\ 75205 Paris Cedex 13, France \\ (e-mail: bassam.fayad@imj-prg.fr) \\ $\ddagger$ Department of Mathematics, KTH, Royal Institute of Technology, \\ SE-100 44 Stockholm, Sweden \\ (e-mail:masha@kth.se)
}

(Received 10 January 2020 and accepted in revised form 8 May 2020)

To our great friend and mentor Anatoly Katok

Abstract. We present examples of nearly integrable analytic Hamiltonian systems with several strong diffusion properties: topological weak mixing and diffusion at all times. These examples are obtained by AbC constructions with several frequencies.

Key words: Hamiltonian systems, diffusion, topological weak mixing, Anosov-Katok method, AbC method

2020 Mathematics Subject Classification: 37J40 (Primary); 37 J39 (Secondary)

\section{Introduction}

KAM theory (after Kolmogrov, Arnol'd, and Moser) states that, under mild non-degeneracy assumptions, Hamiltonian systems close to integrable have their phase space almost completely filled by invariant quasi-periodic tori. Starting from three degrees of freedom for autonomous Hamiltonians the existence of such tori on an energy surface does not prevent the orbits from circulating between the tori inside the surface. Indeed, it was conjectured by Arnol'd that a 'general' Hamiltonian should have a dense orbit on a 'general' energy surface [A]. A large amount of work has been dedicated to proving this conjecture (giving a precise meaning to the word 'general'), but the picture is not yet completely clear, especially when it comes to real analytic Hamiltonians (see for example [BKZ] and references therein).

In his ICM list of problems [H], Herman asks: Can one find an example of a $C^{\infty}$-Hamiltonian $H$ in a small $C^{k}$-neighborhood, $k \geq 2$, of $H_{0}=\|r\|^{2} / 2$ such that on the energy surface $\{H=1\}$ the Hamiltonian flow has a dense orbit? 
A remarkable result in this direction is due to [KZZ]: they present an example of a Hamiltonian $H$ of the form $H(\theta, r)=(\langle r, r\rangle / 2)+h(r, \theta) \in C^{\infty}$ with a trajectory dense in a subset of the energy surface of large measure.

Here we present examples, with a degenerate integrable part, but with even more chaotic behavior for the perturbed system. Namely, we show that in a class of perturbations of rotators, the generic Hamiltonian is topologically weakly mixing on every energy surface. We also give examples of perturbed rotators for which the dynamics is diffusive at all times.

We now give the exact definitions of these properties and state our results precisely.

Definition 1.1. (Topological weak mixing) We say that the flow $\Phi_{H}^{t}$ is topologically weakly mixing if there exists a sequence $\left(t_{n}\right)_{n \in \mathbb{N}}$ such that for any two open sets $A, B$ on the same (arbitrarily chosen) energy surface $E_{H}(c)=\{(\theta, r) \mid H(\theta, r)=c\}$, there exists $N=N(A, B)$ such that $\Phi_{H}^{t_{n}}(A) \cap B \neq \emptyset$ for all $n \geq N$.

Definition 1.2. (Diffusion at all times) We say that the flow $\Phi_{H}^{t}$ exhibits diffusion at all times if for any open set $A \subset \mathbb{T}^{d} \times \mathbb{R}^{d}$ and any $R \geq 0$, there exists $T=T(A, R)$ such that $\left|\pi_{r}\left(\Phi_{H}^{t}(A)\right)\right|>R$ for all $t \geq T$. Here $\pi_{r}$ denotes the projection onto the $r$-variables.

Given $\rho>0$, denote by $C_{\rho}^{\omega}$ the space of bounded real functions on $\mathbb{R}^{d} \times \mathbb{R}^{d}$, that are $\mathbb{Z}^{d}$-periodic in the first $d$-vector of components, and can be extended to holomorphic functions on $D_{\rho}=\left\{(\theta, r) \in\left(\mathbb{C}^{d}, \mathbb{C}^{d}\right) \mid \max _{j}\left\{\left|\theta_{j}\right|,\left|r_{j}\right|, j=1, \ldots d\right\} \leq \rho\right\}$ as $\mathbb{Z}^{d}$-periodic with respect to the real part, with the norm $\|f\|_{\rho}=\sup \left\{|f(\theta, r)|,(\theta, r) \in D_{\rho}\right\}$.

Let $\mathcal{O}_{\rho, \delta}^{\omega}=\left\{f \in C_{\rho}^{\omega} \mid\|f\|_{\rho}<\delta\right\}$ be a neighborhood of zero in the space of analytic functions with the above norm. This is a Baire space. Fixing $\rho=1$ without loss of generality, we write $\|f\|=\|f\|_{1}$, and $\mathcal{O}_{\delta}^{\omega}$ for $\mathcal{O}_{1, \delta}^{\omega}$.

THEOREM A. There exists a dense set $Y \subset \mathbb{R}^{d}, d \geq 3$, ( $Y$ continuum) such that for any $\omega \in Y$, for any $\delta>0$, there exists a real analytic function $h: \mathbb{T}^{d} \rightarrow \mathbb{R}$, such that $h \in \mathcal{O}_{\delta}^{\omega}$, with the following property: the Hamiltonian flow $\Phi_{H}^{t}(\theta, r)$ defined by the Hamiltonian

$$
H=\langle r, \omega\rangle+h(\theta)
$$

exhibits diffusion at all times.

By a generic set in this paper we mean a dense $G_{\delta}$ set.

THEOREM B. For any $d \geq 3$, for a generic $\omega \in \mathbb{R}^{d}$, any $\delta>0$ and generic $h_{1}(\theta), h_{2}(\theta) \in$ $\mathcal{O}_{\delta}^{\omega}$, the flow $\Phi_{H}^{t}$ of the Hamiltonian

$$
H(\theta, r)=\frac{1}{1+h_{1}(\theta)}\left(\langle r, \omega\rangle+h_{2}(\theta)\right)
$$

is topologically weakly mixing.

Note that the energy surface in the statement above is unbounded, which drove us to treat the topological version of weak mixing rather than the classical notion.

Diffusion at all times shows that the rigidity property of the rotator (convergence of the dynamics to identity along a subsequence of times) can be destroyed on all energy surfaces 
by a small perturbation. However, our examples are not topologically mixing, and it would be very interesting to produce examples that are topologically mixing on energy surfaces.

An interesting question concerns the possibility of similar examples in a neighborhood of an elliptic equilibrium. If the components of the frequency vector at the equilibrium are all of the same sign, the energy surfaces are bounded. We hope that the methods of [FS] can be used to construct smooth examples of topologically weakly mixing Hamiltonians in this context as well.

From the form of the perturbations of the rotator in Theorems A and B, we can see that the Hamiltonians that we construct are, in fact, in the closure of Hamiltonians that are conjugate to the rotator. Just like reparametrizations of linear flows on the torus, or abelian skew products above them, our constructions can thus be viewed a posteriori as particular instances of the Approximation by Conjugation (AbC) method [AK], which is also called the AK-method in reference to Dmitry Anosov and Anatoly Katok who first introduced it. The method was already used in the Hamiltonian context by Katok in [K2] to show the existence of integrable degenerate Hamiltonians with some particular Liouville frequencies and bounded energy surfaces that can be smoothly perturbed to become ergodic on the energy surfaces. Subsequent constructions that use the AbC method with several frequencies appeared in [EFK, FS, FF] to discuss the stability of elliptic equilibria and invariant tori, in particular those with Diophantine frequency vectors. The examples of [K2] were constructed following the usual AK-method with successive conjugations of a circle action, which gives $C^{\infty}$ flows that are rigid in the sense that the dynamics converge to identity along a subsequence of times. In our constructions, we bypass the smoothness limitation and the rigidity of the perturbed dynamics by resorting to the reparametrization technique of translation flows in dimensions larger than 3 used in [F]. This technique exploits the Liouville phenomenon in several directions [Y] to avoid the Denjoy-Koksma cancellations that appear in dimension two $[\mathrm{K} 1, \mathbf{K o c}]$.

\section{Notation and definitions}

To alleviate the notation, we will give the proofs for $d=3$ since there is no difference at all in the proof of the general case.

\subsection{General notation.}

- For a vector $r$, its components are denoted by $r_{j}, j=1,2,3$; for a vector $r_{0} \in \mathbb{R}^{3}$, we denote its components by $r_{0, j}, j=1,2,3$.

- For $a \in \mathbb{R}$ and $l>0$, we denote

$$
I(a, l)=a+[0, l]=[a, a+l] .
$$

- $\quad$ For a set $S$ in the phase space, let $\pi_{r}(S)$ stand for the orthogonal projection of $S$ onto the space of actions ( $r$-space). In a similar way, introduce notation $\pi_{\theta}(S), \pi_{r_{j}}(S)$, $\pi_{\theta_{j}}(S)$ for $j=1, \ldots, d$.

- When we write that $p / q$ is a rational number or $(p / q) \in \mathbb{Q}$, we assume that $q \in \mathbb{N}$, $q \geq 1, p \in \mathbb{Z}$, and the numbers $p$ and $q$ are relatively prime.

- Let $\omega=\left(\omega_{1}, \omega_{2}, \omega_{3}\right) \in \mathbb{R}^{3}$ be a rationally independent vector. Then, in particular, $\omega_{3} \neq 0$, and we can rewrite $\omega=\omega_{3}\left(\left(\omega_{1} / \omega_{3}\right),\left(\omega_{2} / \omega_{3}\right), 1\right)$. Without loss of 
generality, the constructions are performed under the assumption that $\omega$ is normalized: $\omega=\left(\alpha, \alpha^{\prime}, 1\right)$.

- Denote $H_{0}(\theta, r)=\langle\omega, r\rangle=\sum_{j=1}^{3} \omega_{j} r_{j}$.

- We use $\Phi_{H}^{t}(\cdot)$ to denote the flow map at time $t$, defined by the Hamiltonian $H$.

- Fix $c \in \mathbb{R}$ and consider an energy surface $E_{H}(c)=\{(\theta, r) \mid H(\theta, r)=c\}$. In our constructions, $\left\|H-H_{0}\right\|_{1}$ is small, so $E_{H}(c)$ is uniformly close to $E_{H_{0}}(c)=\{r \mid$ $\langle r, \omega\rangle=c\} \times \mathbb{T}^{3}$.

\subsection{Arithmetic reminders. Yoccoz pairs of frequencies. Denote}

$$
\||k \alpha|\|=\inf _{p \in \mathbb{Z}}|k \alpha+p| .
$$

For an irrational number $\alpha$ there exists a sequence of rational numbers $\left(p_{n} / q_{n}\right)_{n \geq 1}$, called the convergents of $\alpha$ such that

$$
\left\|\left|q _ { n - 1 } \alpha \left\||<\|k \alpha\|| \text { for all } k<q_{n},\right.\right.\right.
$$

and for any $n$

$$
\frac{1}{q_{n}+q_{n+1}} \leq(-1)^{n}\left(q_{n} \alpha-p_{n}\right) \leq \frac{1}{q_{n+1}}
$$

Definition 2.1. Let $\omega=\left(\alpha, \alpha^{\prime}, 1\right) \in \mathbb{R}^{3}$ where $\alpha$ and $\alpha^{\prime}$ are irrational real numbers with the corresponding sequences of convergents $\left(p_{n} / q_{n}\right)_{n \geq 1}$ and $\left(p_{n}^{\prime} / q_{n}^{\prime}\right)_{n \geq 1}$. We say that $\omega=$ $\left(\alpha, \alpha^{\prime}, 1\right) \in Y$ if for all $n=0, \ldots, \infty$, the denominators of the convergents of $\alpha$ and $\alpha^{\prime}$, respectively, satisfy:

$$
e^{q_{n}} \leq q_{n}^{\prime} / 4, \quad e^{q_{n}^{\prime}} \leq q_{n+1} / 4
$$

By [Y], the set $Y$ is non-empty, of cardinality continuum. This is the set of frequencies used in Theorem A.

2.3. Intervals and rectangles in an energy surface. Here we describe the standard sets used in the construction. In particular, intervals are defined to be small one-dimensional curves that lie in a given energy surface and whose projection onto the five-dimensional space $\left(\theta, r_{1}, r_{2}\right)$ is a linear segment parallel either to the $\theta_{1}$ axis or to the $\theta_{2}$ axis. The coordinate $r_{3}$ is defined by the requirement that the curve lies in the energy surface. More precisely, we have the following.

- Given $s_{0}=\left(\theta_{0}, r_{0}\right)$ and $l>0$, define the intervals

$$
\begin{aligned}
& J^{(1)}\left(s_{0}, l\right) \\
& =\left\{(\theta, r) \in I\left(\theta_{0,1}, l\right) \times\left\{\theta_{0,2}\right\} \times\left\{\theta_{0,3}\right\} \times\left\{r_{0,1}\right\} \times\left\{r_{0,2}\right\} \times \mathbb{R} \mid H(\theta, r)=H\left(\theta_{0}, r_{0}\right)\right\},
\end{aligned}
$$

and

$$
\begin{aligned}
& J^{(2)}\left(s_{0}, l\right) \\
& =\left\{(\theta, r) \in\left\{\theta_{0,1}\right\} \times I\left(\theta_{0,2}, l\right) \times\left\{\theta_{0,3}\right\} \times\left\{r_{0,1}\right\} \times\left\{r_{0,2}\right\} \times \mathbb{R} \mid H(\theta, r)=H\left(\theta_{0}, r_{0}\right)\right\} .
\end{aligned}
$$


- For any $s_{0}=\left(\theta_{0}, r_{0}\right), l_{1}>0$ and $l_{2}>0$, define the rectangle

$$
\begin{aligned}
& R\left(s_{0}, l_{1}, l_{2}\right) \\
& =\left\{(\theta, r) \in I\left(\theta_{0,1}, l_{1}\right) \times I\left(\theta_{0,2}, l_{2}\right) \times\left\{\theta_{0,3}\right\} \times\left\{r_{0,1}\right\} \times\left\{r_{0,2}\right\} \times \mathbb{R} \mid H(\theta, r)\right. \\
& \left.=H\left(\theta_{0}, r_{0}\right)\right\} .
\end{aligned}
$$

As before, the projection of $R\left(\theta_{0}, r_{0}, l_{1}, l_{2}\right)$ onto the space $\left(\theta, r_{1}, r_{2}\right)$ is a flat rectangle parallel to the $\left(\theta_{1}, \theta_{2}\right)$-plane; $r_{3}$ is chosen so that $R\left(\theta_{0}, r_{0}, l_{1}, l_{2}\right) \subset E_{H}(c)$.

We say that the size of the rectangle $R\left(\theta_{0}, r_{0}, l_{1}, l_{2}\right)$ is $l_{1} \times l_{2}$.

- Given $n$ and $s_{0}=\left(\theta_{0}, r_{0}\right)$, a box $B_{n}\left(s_{0}\right) \subset E_{H}(c)$ is defined by

$$
\begin{aligned}
B_{n}\left(s_{0}\right)= & \left\{(\theta, r) \mid \theta_{j} \in I\left(\theta_{0, j}, \frac{1}{n}\right), j=1,2,3,\right. \\
& \left.r_{j} \in I\left(r_{0, j}, \frac{1}{n}\right), j=1,2, H(\theta, r)=H\left(\theta_{0}, r_{0}\right)\right\} .
\end{aligned}
$$

These sets, having full dimension in $E_{H}(c)$, will be used as test sets: in particular, to prove Theorem B, we will show that at certain times $t_{n}$, the image of any rectangle $R_{n} \subset E_{H}(c)$ intersects each box $B_{n} \subset E_{H}(c)$. To do so, we need the notion of stretching.

Definition 2.2. Given positive $l, L$, and $t$, we say that the flow map $\Phi_{H}^{t}$ is $(1, l, L)$-stretching if for any interval $J^{(1)}=J^{(1)}\left(\theta_{0}, r_{0}, l\right)$ with $\left|r_{0}\right| \leq L / 10$ we have

$$
\pi_{r_{1}}\left(\Phi_{H}^{t}\left(J^{(1)}\right)\right) \supset[-L, L]
$$

and the map $(\theta, r) \mapsto \pi_{r_{1}}\left(\Phi_{H}^{t}(\theta, r)\right)$ is independent of $\theta_{2}$ where $\theta=\left(\theta_{1}, \theta_{2}, \theta_{3}\right)$. Analogously, we say that the flow map $\Phi_{H}^{t}$ is $(2, l, L)$-stretching if for any interval $J^{(2)}=$ $J^{(2)}\left(\theta_{0}, r_{0}, l\right)$ with $\left|r_{0}\right| \leq L / 10$ we have

$$
\pi_{r_{2}}\left(\Phi_{H}^{t}\left(J^{(2)}\right)\right) \supset[-L, L],
$$

and the map $(\theta, r) \mapsto \pi_{r_{2}}\left(\Phi_{H}^{t}(\theta, r)\right)$ is independent of $\theta_{1}$.

\section{Proofs of the main theorems}

Here we prove the main theorems modulo the technical statements, whose demonstration is deferred to the next section.

3.1. The construction for Theorem $A$. Let us fix an arbitrary vector $\left(\alpha, \alpha^{\prime}, 1\right) \in Y$ with $\left(p_{n} / q_{n}\right)_{n \geq 1}$ and $\left(p_{n}^{\prime} / q_{n}^{\prime}\right)_{n \geq 1}$ being the corresponding sequences of convergents. Let

$$
\begin{aligned}
h(\theta) & =-\sum_{n=1}^{\infty} h_{n}(\theta)-\sum_{n=1}^{\infty} h_{n}^{\prime}(\theta), \\
h_{n}(\theta) & =e^{-q_{n}} \cos 2 \pi\left(q_{n} \theta_{1}-p_{n} \theta_{3}\right), \quad h_{n}^{\prime}(\theta)=e^{-q_{n}^{\prime}} \cos 2 \pi\left(q_{n}^{\prime} \theta_{2}-p_{n}^{\prime} \theta_{3}\right) .
\end{aligned}
$$


Theorem A follows from the result below.

THEOREM 3.1. For any $\omega \in Y$, for $h$ as in (3.1), the Hamiltonian flow $\Phi_{H}^{t}(\theta, r)$ defined by the Hamiltonian

$$
H=\langle r, \omega\rangle+h(\theta)
$$

exhibits diffusion at all times.

Remark 3.2. This Hamiltonian can be seen as a limit of an Anosov-Katok-type construction, that is, it has the form

$$
H=\lim H^{(n)}, \quad H^{(n)}=H_{0} \circ \Psi_{n} \circ \cdots \circ \Psi_{1},
$$

where $\Psi_{i}$ are symplectic analytic coordinate changes.

The proof of Theorem 3.1 relies on the following proposition that is proved in $\$ 4.1$.

Proposition 3.3. For any $\omega \in Y$, for $h$ as in (3.1), the Hamiltonian flow $\Phi_{H}^{t}(\theta, r)$ defined by the Hamiltonian

$$
H=\langle r, \omega\rangle+h(\theta)
$$

satisfies for each $n$ :

(a) for all $t \in\left[e^{q_{n}}, q_{n+1} / 4\right]$, $\Phi_{H}^{t}$ is $\left(1,1 / q_{n}, q_{n}\right)$-stretching;

(b) for all $t \in\left[e^{q_{n}^{\prime}}, q_{n+1}^{\prime} / 4\right]$, $\Phi_{H}^{t}$ is $\left(2,1 / q_{n}^{\prime}, q_{n}^{\prime}\right)$-stretching.

Here we show how this proposition implies Theorem 3.1.

Proof of Theorem 3.1. As the sequences $\left(q_{n}\right)$ and $\left(q_{n}^{\prime}\right)$ satisfy (2.2), the union of the intervals $\bigcup_{n>N}\left[e^{q_{n}}, q_{n+1} / 4\right] \cup\left[e^{q_{n}^{\prime}}, q_{n+1}^{\prime} / 4\right]$ contains the half-line $t>e^{q_{N}}$. Proposition 3.3 implies that for each $t \in\left[e^{q_{n}}, q_{n+1} / 4\right], \Phi_{H}^{t}$ stretches small rectangles in the direction of $r_{1}$ with a large factor, and for each $t \in\left[e^{q_{n}^{\prime}}, q_{n+1}^{\prime} / 4\right], \Phi_{H}^{t}$ stretches small rectangles in the direction of $r_{2}$.

Hence, $\Phi_{H}^{t}$ exhibits stretching with an increasingly strong factor as $t \rightarrow \infty$, in at least one of the two directions $r_{1}$ and $r_{2}$. This implies the conclusion of Theorem 3.1.

3.2. The construction for Theorem $B$. Consider $\omega=\left(\alpha, \alpha^{\prime}, 1\right)$, and suppose that there exist sequences $\left(p_{n} / q_{n}\right)_{n \geq 1}$ and $\left(p_{n}^{\prime} / q_{n}^{\prime}\right)_{n \geq 1}$ such that

$$
q_{n}^{4} \leq q_{n}^{\prime}, \quad\left|q_{n} \alpha-p_{n}\right| \leq e^{-2 q_{n}^{\prime}}, \quad\left|q_{n}^{\prime} \alpha^{\prime}-p_{n}^{\prime}\right| \leq e^{-q_{n}^{\prime}} .
$$

We start by observing that the set $S$ of pairs $\left(\alpha, \alpha^{\prime}\right)$ with this assumption contains a generic set in $\mathbb{R}^{2}$. This implies, of course, that the set of numbers $\omega=\left(\omega_{1}, \omega_{2}, \omega_{3}\right)=\omega_{3}\left(\alpha, \alpha^{\prime}, 1\right)$ such that $\left(\alpha, \alpha^{\prime}\right) \in S$ and $\omega_{3} \in \mathbb{R}$, is generic in $\mathbb{R}^{3}$.

LEMMA 3.4. There exists a generic (dense $G_{\delta}$ ) set $\hat{S} \subset S \subset \mathbb{R}^{2}$ of pairs $\left(\alpha, \alpha^{\prime}\right)$ satisfying the following: there exist sequences $\left(p_{n} / q_{n}\right)_{n \geq 1}$ and $\left(p_{n}^{\prime} / q_{n}^{\prime}\right)_{n \geq 1}$ of rational numbers such that estimate (3.2) holds for all $n$. 
Proof. We want to describe the set $S$ of pairs $\left(\alpha, \alpha^{\prime}\right)$ such that for any $N$ there exist $p / q$ and $p^{\prime} / q^{\prime} \in \mathbb{Q}$ such that $q>N, q^{\prime} \geq q^{4}, p, p^{\prime} \in \mathbb{Z}$, and

$$
|\alpha-p / q| \leq e^{-2 q^{\prime}} / q, \quad\left|\alpha^{\prime}-p^{\prime} / q^{\prime}\right| \leq e^{-q^{\prime}} / q^{\prime} .
$$

The set $S$ contains the following set $\hat{S}$ :

$$
\bigcap_{N \geq 1}\left(\bigcup_{q \geq N} \bigcup_{p \in \mathbb{Z}} \bigcup_{q^{\prime} \geq q^{4}} \bigcup_{p^{\prime} \in \mathbb{Z}}\left(\frac{p}{q}-\frac{e^{-2 q}}{q}, \frac{p}{q}+\frac{e^{-2 q}}{q}\right) \times \leq\left(\frac{p^{\prime}}{q^{\prime}}-\frac{e^{-q^{\prime}}}{q^{\prime}}, \frac{p^{\prime}}{q^{\prime}}+\frac{e^{-q^{\prime}}}{q^{\prime}}\right)\right),
$$

which is a countable intersection (in $N$ ) of open dense sets.

Here we present an explicit example of a Hamiltonian whose flow is topologically weakly mixing. It is easy to see that such examples can be produced arbitrarily close to $H_{0}$. From this, the genericity of Hamiltonians with the weak mixing property is obtained in the standard way.

Having fixed $\omega \in \mathbb{R}^{3}$ as in (3.2), let

$$
\phi(\theta)=1+\sum_{n=1}^{\infty} q_{n} e^{-q_{n}^{\prime}} \cos 2 \pi\left(q_{n} \theta_{1}-p_{n} \theta_{3}\right)
$$

and for $\kappa_{n}:=q_{n}^{2}$, introduce

$$
\begin{aligned}
\tilde{h}(\theta) & =-\sum_{n=1}^{\infty} \tilde{h}_{n}(\theta)-\sum_{n=1}^{\infty} \tilde{h}_{n}^{\prime}(\theta), \\
\tilde{h}_{n}(\theta) & =\kappa_{n} e^{-q_{n}^{\prime}} \cos 2 \pi \kappa_{n}\left(q_{n} \theta_{1}-p_{n} \theta_{3}\right), \quad \tilde{h}_{n}^{\prime}(\theta)=e^{-q_{n}^{\prime}} \cos 2 \pi\left(q_{n}^{\prime} \theta_{2}-p_{n}^{\prime} \theta_{3}\right) .
\end{aligned}
$$

Note that because $\left\|\tilde{h}_{n}\right\|_{1} \leq \kappa_{n} e^{-q_{n}^{\prime}} e^{2 \pi \kappa_{n}\left(q_{n}+p_{n}\right)} \leq q_{n}^{2} e^{4 \pi q_{n}^{3}-q_{n}^{\prime}}$, assumption $q_{n}^{4} \leq q_{n}^{\prime}$ implies that $\left\|\sum_{n=1}^{\infty} \tilde{h}_{n}(\theta)\right\|_{1}<\infty$. Clearly, this implies that $\|\tilde{h}(\theta)\|_{1}<\infty$.

THEOREM 3.5. For $\omega$ as in (3.2), $\phi$ as in (3.3), $\tilde{h}$ as in (3.4), the Hamiltonian flow $\Phi_{\tilde{H}}^{t}(\theta, r)$ defined by

$$
\tilde{H}=\frac{1}{\phi(\theta)}(\langle r, \omega\rangle+\tilde{h}(\theta))
$$

is topologically weakly mixing.

More precisely, for $t_{n}=e^{q_{n}^{\prime}}, n \geq 1$, we have: for any two open sets $A$ and $B$ on the same energy surface there exists $N=N(A, B)$ such that

$$
\Phi_{\tilde{H}}^{t_{n}}(A) \cap B \neq \varnothing \quad \text { for all } n \geq N .
$$

Assuming Theorem 3.5, we show how it yields Theorem B.

Proof of Theorem B. It follows from classical arguments (cf. [Ha]) that weak mixing for the flows as in Theorem B holds for a $G_{\delta}$-set of functions $\left(h_{1}, h_{2}\right) \in \mathcal{O}_{\delta}^{\omega}(0)^{2}$. It is left to show the density of weak mixing for $\left(h_{1}, h_{2}\right) \in \mathcal{O}_{\delta}^{\omega}(0)^{2}$ for a fixed $\delta$. To do this, note that for any $\epsilon>0$, both $(\phi(\theta)-1)$ and $\tilde{h}(\theta)$ can be chosen $\epsilon$-close to zero in the fixed norm: it is enough to choose $q_{1}$ large enough. Moreover, from the proof of Theorem 3.5 it follows that the same result holds true if we change $\phi(\theta)$ and $\tilde{h}(\theta)$ by $\phi(\theta)+P(\theta) \in \mathcal{O}_{\delta}^{\omega}(0)$ and 
$\tilde{h}(\theta)+Q(\theta) \in \mathcal{O}_{\delta}^{\omega}(0)$ with $P$ and $Q$ trigonometric polynomials. This implies the density of the weak mixing property.

The proof of Theorem 3.5 relies on the following two propositions that are proved in $\S \S 4.1$ and 4.2 , respectively.

Proposition 3.6. For $\omega$ as in (3.2), $\phi$ as in (3.3), $\tilde{h}$ as in (3.4), $\tilde{H}$ as in (3.5), and $t_{n}=e^{q_{n}^{\prime}}$, we have:

(a) $\Phi_{\tilde{H}}^{t_{n}}$ is $\left(1,1 / q_{n}^{3}, q_{n}\right)$-stretching;

(b) $\Phi_{\tilde{H}}^{t_{n}}$ is $\left(2,1 / q_{n}^{\prime}, q_{n}^{\prime}\right)$-stretching.

Proposition 3.7. For $\omega$ as in (3.2), $\phi$ as in (3.3), $\tilde{h}$ as in (3.4), the Hamiltonian flow $\Phi_{\tilde{H}}^{t}(\theta, r)$ defined by

$$
\tilde{H}=\frac{1}{\phi(\theta)}(\langle r, \omega\rangle+\tilde{h}(\theta))
$$

satisfies for $t_{n}=e^{q_{n}^{\prime}}$ the following. For any rectangle $R_{n}:=R\left(\theta_{0}, r_{0}, 1 / q_{n}, 1 / q_{n}\right)$ with $\left|r_{0}\right| \leq n$ and any box $B_{n}$ (see notation in $\$ 2.3$ ) there exists a rectangle $R_{n}^{\prime} \subset R_{n}$ of size $1 / q_{n}^{3} \times 1 / q_{n}^{3}$ such that

$$
\pi_{\theta}\left(\Phi_{\tilde{H}}^{t_{n}}\left(R_{n}^{\prime}\right)\right) \subset \pi_{\theta}\left(B_{n}\right)
$$

Proof of Theorem 3.5. Fix $R_{n}$ and $B_{n}$ as previously.

By Proposition 3.7, there exists a rectangle $R_{n}^{\prime} \subset R_{n}$ of size $1 / q_{n}^{3} \times 1 / q_{n}^{3}$ such that

$$
\pi_{\theta}\left(\Phi_{\tilde{H}}^{t_{n}}\left(R_{n}^{\prime}\right)\right) \subset \pi_{\theta}\left(B_{n}\right) .
$$

By Proposition 3.6, we can find a rectangle $\bar{R}_{n} \subset R_{n}^{\prime}$ such that

$$
\pi_{r}\left(\Phi_{\tilde{H}}^{t_{n}}\left(\bar{R}_{n}\right)\right) \subset \pi_{r}\left(B_{n}\right) .
$$

Hence

$$
\left.\Phi_{\tilde{H}}^{t_{n}}\left(\bar{R}_{n}\right)\right) \subset B_{n}
$$

and the proof is finished.

4. Stretching

4.1. Stretching in the action directions.

LEMMA 4.1. Let $p / q,\left(p^{\prime} / q^{\prime}\right) \in \mathbb{Q}$ and $\omega=\left(\alpha, \alpha^{\prime}, 1\right)$ and $a_{q}, b_{q^{\prime}}$ satisfy

$$
e^{-q} \geq a_{q} \geq 4|q \alpha-p|, \quad e^{-q^{\prime}} \geq b_{q^{\prime}} \geq 4\left|q^{\prime} \alpha^{\prime}-p^{\prime}\right| .
$$

Define

$$
H(\theta, r)=\langle r, \omega\rangle-a_{q} \cos 2 \pi\left(q \theta_{1}-p \theta_{3}\right)-b_{q^{\prime}} \cos 2 \pi\left(q^{\prime} \theta_{2}-p^{\prime} \theta_{3}\right) .
$$

Then the following hold:

(a) for each $t \in\left[a_{q}^{-1}, 1 /(4|q \alpha-p|)\right]$ the flow map $\Phi_{H}^{t}$ is $(1,1 / q, 2 q)$ stretching;

(b) for each $t \in\left[b_{q^{\prime}}^{-1}, 1 /\left(4\left|q^{\prime} \alpha^{\prime}-p^{\prime}\right|\right)\right]$, the flow map $\Phi_{H}^{t}$ is $\left(2,1 / q^{\prime}, 2 q^{\prime}\right)$ stretching. 
Proof of Lemma 4.1. The Hamiltonian $H$ defines the following system of equations (we omit $r_{3}$ from the considerations):

$$
\left\{\begin{array}{l}
\dot{\theta}=\omega, \\
\dot{r}_{1}=-2 \pi q a_{q} \sin 2 \pi\left(q \theta_{1}-p \theta_{3}\right), \\
\dot{r}_{2}=-2 \pi q^{\prime} b_{q^{\prime}} \sin 2 \pi\left(q^{\prime} \theta_{2}-p^{\prime} \theta_{3}\right) .
\end{array}\right.
$$

This system can be integrated explicitly: the solution with initial conditions $(\theta(0), r(0))=$ $\left(\theta_{0}, r_{0}\right)$ satisfies

$$
\left\{\begin{array}{l}
\theta=\theta_{0}+t \omega, \\
r_{1}(t)=c_{1}+\frac{q a_{q}}{q \alpha-p} \cos 2 \pi\left(\left(q \theta_{0,1}-p \theta_{0,3}\right)+t(q \alpha-p)\right), \\
r_{2}(t)=c_{2}+\frac{q^{\prime} b_{q^{\prime}}}{q^{\prime} \alpha^{\prime}-p^{\prime}} \cos 2 \pi\left(\left(q^{\prime} \theta_{0,2}-p^{\prime} \theta_{0,3}\right)+t\left(q^{\prime} \alpha-p^{\prime}\right)\right),
\end{array}\right.
$$

where $c_{k}$ is a constant such that $r_{k}(0)=r_{0, k}, k=1,2$. Note that $r_{1}(t)=\pi_{r_{1}}\left(\Phi_{H}^{t}\left(r_{0}, \theta_{0}\right)\right)$ is independent of $\theta_{0,2}$, and $r_{2}(t)=\pi_{r_{2}}\left(\Phi_{H}^{t}(r, \theta)\right)$ is independent of $\theta_{0,1}$.

Fix an arbitrary $t \in\left[a_{q}^{-1}, 1 /(4(q \alpha-p))\right]$ and $s_{0}=\left(r_{0}, \theta_{0}\right)$ with $\left|r_{0}\right|<q / 10$, and consider the interval $J^{(1)}=J^{(1)}\left(s_{0}, 1 / q\right)$, see notation in $\S 2.3$. Assume $q \alpha-p>0$ (the opposite case is similar). There exists a point $s^{+}=\left(\theta^{+}, r_{0}\right) \in J^{(1)}$ with $\theta^{+}=$ $\left(\theta_{1}^{+}, \theta_{0,2}, \theta_{0,3}\right)$ such that

$$
q \theta_{1}^{+}-p \theta_{03}=-1 / 4 \bmod 1 .
$$

Then $\cos 2 \pi\left(q \theta_{1}^{+}-p \theta_{03}\right)=0$. Consider the trajectory of the above flow with the initial condition $(\theta(0), r(0))=\left(\theta^{+}, r_{0}\right)$. For the first action component this reads: $r_{1}(0)=c_{1}=$ $r_{0,1}$, which implies

$$
\left|c_{1}\right|=\left|r_{0,1}\right| \leq\left|r_{0}\right| \leq q / 10 .
$$

Assumption $t \in\left[a_{q}^{-1}, 1 /(4(q \alpha-p))\right]$ implies, in particular, $(2 \pi t(q \alpha-p)) \in$ $[0, \pi / 2]$. Using the fact that $\sin (x) \geq x / 2$ for all $x \in[0, \pi / 2]$, we obtain

$$
\begin{aligned}
& \frac{q a_{q}}{q \alpha-p} \cos 2 \pi\left(\left(q \theta_{1}^{+}-p \theta_{03}\right)+t(q \alpha-p)\right) \\
& \quad=\frac{q a_{q}}{q \alpha-p} \sin 2 \pi t(q \alpha-p) \geq \pi t q a_{q} \geq 3 q .
\end{aligned}
$$

As $\left|c_{1}\right|<q / 10$, we obtain $\pi_{r_{1}}\left(\Phi_{H}^{t}\left(s^{+}\right)\right)>2 q$.

In the same way, there is a point $s^{-} \in J^{(1)}$ such that $\pi_{r_{1}}\left(\Phi_{H}^{t}\left(s^{-}\right)\right)<-2 q$. The result follows by continuity.

Here we prove Proposition 3.3 that was used for the proof of Theorem 3.1.

Proof of Proposition 3.3. Let us prove statement $(a)$, the second statement is similar. As $q_{n} / p_{n}$ is a convergent of $\alpha$, by (2.1) we have for all $n$ that $q_{n+1} \leq 1 /\left|q_{n} \alpha-p_{n}\right|$. Condition (2.2) implies that $e^{q_{n}} \leq q_{n+1} / 4$, so we have

$$
\left[e^{q_{n}}, q_{n+1} / 4\right] \subset\left[e^{q_{n}}, 1 /\left(4\left|q_{n} \alpha-p_{n}\right|\right)\right] .
$$


Consider $H_{n}(\theta, r)=\langle r, \omega\rangle-h_{n}(\theta)-h_{n}^{\prime}(\theta)$, where $h_{n}, h_{n}^{\prime}$ are defined by (3.1). Fix $r=r_{0}$ with $\left|r_{0}\right|<q / 10$. The first component of $\Phi_{H_{n}}^{t}\left(\theta, r_{0}\right)$, that is, $r_{n, 1}\left(\theta, r_{0}, t\right):=$ $\pi_{r_{1}}\left(\Phi_{H_{n}}^{t}\left(\theta, r_{0}\right)\right)$, is given by

$$
\begin{aligned}
r_{n, 1}\left(\theta, r_{0}, t\right) & =c_{n}\left(\theta, r_{0}\right)+\frac{q_{n} e^{-q_{n}}}{q_{n} \alpha-p_{n}} \cos 2 \pi\left(\left(q_{n} \theta_{1}-p_{n} \theta_{3}\right)+t\left(q_{n} \alpha-p_{n}\right)\right) \\
& :=c_{n}\left(\theta, r_{0}\right)+f_{n}(\theta, t),
\end{aligned}
$$

where $c_{n}\left(\theta, r_{0}\right)$ is such that $r_{n, 1}\left(\theta, r_{0}, 0\right)=r_{0}$. By Lemma 4.1, for each $t \in\left[e^{q_{n}}, q_{n+1} / 4\right]$ the flow map $\Phi_{H_{n}}^{t}$ is $\left(1,1 / q_{n}, 2 q_{n}\right)$ stretching, that is, in any interval $J^{(1)}\left(r_{0}, \theta_{0}, 1 / q_{n}\right)$ with $\left|r_{0}\right| \leq q_{n} / 10$ there are points $s^{+}=\left(r_{0}, \theta^{+}\right)$and $s^{-}=\left(r_{0}, \theta^{-}\right)$such that

$$
r_{n, 1}\left(s^{+}, t\right) \geq 2 q_{n}, \quad r_{n, 1}\left(s^{-}, t\right) \leq-2 q_{n} .
$$

Hence, in particular,

$$
f_{n}\left(\theta^{+}, t\right)-f_{n}\left(\theta^{+}, t\right)=r_{n, 1}\left(s^{+}, t\right)-r_{n, 1}\left(s^{+}, 0\right) \geq 2 q_{n}-q_{n} / 10=1.9 q_{n} .
$$

Let us show that for these $t, \Phi_{H}^{t}$ has the same stretching properties as $\Phi_{H_{n}}^{t}$ (with $2 q_{n}$ replaced by $\left.q_{n}\right)$. The first component of $\Phi_{H}^{t}\left(\theta, r_{0}\right)$, that is, $r_{1}\left(\theta, r_{0}, t\right):=\pi_{r_{1}}\left(\Phi_{H}^{t}\left(\theta, r_{0}\right)\right.$, is given by the formula

$$
\begin{aligned}
r_{1}\left(\theta, r_{0}, t\right) & =c\left(\theta, r_{0}\right)+\sum_{k=1}^{\infty} \frac{q_{k} e^{-q_{k}}}{q_{k} \alpha-p_{k}} \cos 2 \pi\left(\left(q_{k} \theta_{1}-p_{k} \theta_{3}\right)+t\left(q_{k} \alpha-p_{k}\right)\right) \\
& :=c\left(\theta, r_{0}\right)+\sum_{k=1}^{\infty} f_{k}(\theta, t)=c\left(\theta, r_{0}\right)+f_{n}(\theta, t)+C_{n}(\theta, t)+D_{n}(\theta, t),
\end{aligned}
$$

where $C_{n}(\theta, t)=\sum_{k=1}^{n-1} f_{n}(\theta, t), D_{n}(\theta, t)=\sum_{k=n+1}^{\infty} f_{k}(\theta, t)$, and $c\left(\theta, r_{0}\right)$ is a constant such that $r_{1}\left(\theta, r_{0}, 0\right)=r_{0,1}$.

Consider $C_{n}(\theta, t)$. By (2.1), $1 /\left|q_{k} \alpha-p_{k}\right| \leq 2 q_{k+1}$ for all $k \geq 1$, so for any $\theta$ we have

$$
\left|C_{n}(\theta, t)-C_{n}(\theta, 0)\right| \leq 2 \sum_{k=1}^{n-1}\left|\frac{q_{k} e^{-q_{k}}}{q_{k} \alpha-p_{k}}\right| \leq 4 \sum_{k=1}^{n-1} q_{k} e^{-q_{k}} q_{k+1} \leq q_{n} / 100
$$

owing to the growth condition on $q_{k}$.

Now consider $D_{n}(t)$. By (2.1), for any $k \geq 1$ we have $\left|q_{k} \alpha-p_{k}\right| \leq\left(1 / q_{k+1}\right)$. Hence, for any $t \leq q_{n+1}$ we have

$$
\begin{aligned}
\mid D_{n}(\theta, t) & -D_{n}(\theta, 0)|\leq t \sup | D_{n}^{\prime}(\theta, \tilde{t}) \mid \\
& \leq 2 \pi t \sum_{k=n+1}^{\infty}\left|\frac{q_{k} e^{-q_{k}}}{q_{k} \alpha-p_{k}}\right|\left|q_{k} \alpha-p_{k}\right| \leq 2 \pi q_{n+1} \sum_{k=n+1}^{\infty} q_{k} e^{-q_{k}}<1 .
\end{aligned}
$$


This implies that for any $r_{0}$ with $\left|r_{0}\right| \leq q / 10$ we have

$$
\begin{gathered}
r_{1}\left(s^{+}, t\right)-r_{1}\left(s^{+}, 0\right) \geq f_{n}\left(\theta^{+}, t\right)-f_{n}\left(\theta^{+}, t\right)-\left|C_{n}(\theta, t)-C_{n}(\theta, 0)\right| \\
-\left|D_{n}(\theta, t)-D_{n}(\theta, 0)\right| \geq 1.9 q_{n}-0.01 q_{n}-1>1.5 q_{n} .
\end{gathered}
$$

As, by assumption, $\left|r_{1}\left(s^{+}, 0\right)\right|=\left|r_{0}\right| \leq q_{n} / 10$, we obtain

$$
r_{1}\left(s^{+}, t\right) \geq q_{n} .
$$

By the same argument, $r_{1}\left(s^{-}, t\right) \leq-q_{n}$. Thus, $\Phi_{H}^{t}$ is $\left(1,1 / q_{n}, q_{n}\right)$ stretching.

In the following, we prove an analog of Proposition 3.3 for the Hamiltonian $\tilde{H}$ of Theorem 3.5. To begin with, note that our choice of $\phi$ and $\tilde{h}$ implies that the Hamiltonian system of $\tilde{H}$ has a particularly simple form.

LeMmA 4.2. For $\omega$ as in (3.2), $\phi$ as in (3.3), $\tilde{h}$ as in (3.4), the Hamiltonian flow $\Phi_{\tilde{H}}^{t}(\theta, r)$ defined by

$$
\tilde{H}=\frac{1}{\phi(\theta)}(\langle r, \omega\rangle+\tilde{h}(\theta))
$$

satisfies

$$
\left\{\begin{array}{l}
\dot{\theta}=\frac{1}{\phi(\theta)} \omega \\
\dot{r}_{1}=\frac{-2 \pi}{\phi(\theta)} \sum_{n=1}^{\infty} q_{n}\left(C q_{n} e^{-q_{n}^{\prime}} \sin 2 \pi\left(q_{n} \theta_{1}-p_{n} \theta_{3}\right)+\kappa_{n}^{2} e^{-q_{n}^{\prime}} \sin 2 \pi \kappa_{n}\left(q_{n} \theta_{1}-p_{n} \theta_{3}\right)\right) \\
\dot{r}_{2}=\frac{-2 \pi}{\phi(\theta)} \sum_{n=1}^{\infty} q_{n}^{\prime} e^{-q_{n}^{\prime}} \sin 2 \pi\left(q_{n}^{\prime} \theta_{2}-p_{n}^{\prime} \theta_{3}\right),
\end{array}\right.
$$

where $C=\tilde{H}(\theta, r)$. We omit the expression for $r_{3}$ because it is not used in the following.

Proof of Lemma 4.2. Recall that the value of $\tilde{H}(\theta, r)=1 / \phi(\theta)(\langle r, \omega\rangle+\tilde{h}(\theta)):=C$ is constant on the solutions of the corresponding system of equations. For $j=1,2$ we have $\dot{r}_{j}=-\partial_{\theta_{j}} \tilde{H}$, where

$$
\partial_{\theta_{j}} \tilde{H}=-\frac{1}{\phi^{2}} \partial_{\theta_{j}} \phi(\langle r, \omega\rangle+\tilde{h})+\frac{1}{\phi} \partial_{\theta_{j}} \tilde{h}=-\frac{1}{\phi}\left(C \partial_{\theta_{j}} \phi-\partial_{\theta_{j}} \tilde{h}\right) .
$$

Explicit substitution finishes the proof.

In the next lemma (which is an analog of Lemma 4.1) we study the action components of the above system in a simplified form: we consider only the $n$th term in the sums above. The study of the angle components is postponed to Proposition 3.7.

Lemma 4.3. Let $\omega=\left(\alpha, \alpha^{\prime}, 1\right)$, where $\alpha$ and $\alpha^{\prime}$ are irrational. Assume that there exist rational numbers $p / q$ and $p^{\prime} / q^{\prime}$ satisfying (3.2) with $q_{n}$ and $q_{n}^{\prime}$ replaced by $q$ and $q^{\prime}$, respectively. Let $\phi(\theta): \mathbb{T}^{3} \mapsto \mathbb{R}$ be a smooth function satisfying $3 / 4 \leq|\phi(\theta)| \leq 2$ for all $\theta \in \mathbb{T}^{3}$. Denote $\kappa=q^{2}$, take any $C \in \mathbb{R}$ with $|C| \leq q^{1 / 2}$, and $r_{0} \in \mathbb{R}^{3}$ with $\left|r_{0}\right| \leq q / 10$, 
and let $\Phi_{S}^{t}\left(\theta_{0}, r_{0}\right)$ be the flow of the system

$$
\left\{\begin{array}{l}
\dot{\theta}=\frac{1}{\phi(\theta)} \omega \\
\dot{r}_{1}=\frac{-2 \pi}{\phi(\theta)} q\left(C q e^{-q^{\prime}} \sin 2 \pi\left(q \theta_{1}-p \theta_{3}\right)+\kappa^{2} e^{-q^{\prime}} \sin 2 \pi \kappa\left(q \theta_{1}-p \theta_{3}\right)\right) \\
\dot{r}_{2}=\frac{-2 \pi}{\phi(\theta)} q^{\prime} e^{-q^{\prime}} \sin 2 \pi\left(q^{\prime} \theta_{2}-p^{\prime} \theta_{3}\right)
\end{array}\right.
$$

with initial conditions $\Phi_{S}^{0}\left(\theta_{0}, r_{0}\right)=\left(\theta_{0}, r_{0}\right)$. Then for $t=e^{q^{\prime}}$ the following hold:

(a) $\Phi_{S}^{t}$ is $(1,1 / q \kappa, 2 q)$-stretching;

(b) $\Phi_{S}^{t}$ is $\left(2,1 / q^{\prime}, 2 q^{\prime}\right)$-stretching.

Proof of Lemma 4.3. The proof is analogous to that of Lemma 4.1. The only difference is that in this case $\dot{\theta}(t)$ is not constant. Let us study the $r_{1}$-component of $\Phi_{S}^{t}\left(\theta_{0}, r_{0}\right)$ (the analysis of $r_{2}(t)$ is similar). Fix an arbitrary $\left(\theta_{0}, r_{0}\right)$ with $\left|r_{0}\right| \leq q / 10$ and let $\tilde{\boldsymbol{J}}^{(1)}=$ $J^{(1)}\left(\theta_{0}, r_{0}, 1 / \kappa q\right)$.

As $3 / 4 \leq|\phi(\theta)| \leq 2$, the mean value theorem implies that the angle variables satisfy

$$
\theta\left(\theta_{0} ; t\right)=\theta_{0}+t \xi\left(\theta_{0}, t\right) \omega
$$

where $3 / 4 \leq|\xi(\theta, t)| \leq 2$ for all $\theta, t$.

Given an initial condition $r_{1}\left(\theta, r_{0}, 0\right)=r_{0,1}$, the system defines

$$
\begin{aligned}
r_{1}\left(\theta, r_{0} ; t\right)= & c_{1}+\frac{C q^{2} e^{-q^{\prime}}}{q \alpha-p} \cos 2 \pi\left(q \theta_{1}(\theta ; t)-p \theta_{3}(\theta ; t)\right) \\
& +\frac{q \kappa e^{-q^{\prime}}}{q \alpha-p} \cos 2 \pi \kappa\left(q \theta_{1}(\theta ; t)-p \theta_{3}(\theta ; t)\right) \\
& :=c_{1}\left(\theta, r_{0}\right)+g_{1}\left(\theta, r_{0} ; t\right)+g_{2}\left(\theta, r_{0} ; t\right),
\end{aligned}
$$

where $c_{1}\left(\theta, r_{0}\right)$ is the constant such that $r_{1}\left(\theta, r_{0}, 0\right)=r_{0,1}$. Note that $g_{2}\left(\theta_{0}, r_{0}, t\right)$ is the leading term in the above expression. Assume that $q \alpha-p>0$, the opposite case is similar. Clearly, there exists a point $s^{+}=\left(\theta^{+}, r_{0}\right) \in \tilde{J}^{(1)}$ with $\theta^{+}=\left(\theta_{1}^{+}, \theta_{02}, \theta_{03}\right)$ such that

$$
\kappa\left(q \theta_{1}^{+}-p \theta_{03}\right)=-1 / 4 \bmod 1 .
$$

We show that $r_{1}\left(\theta^{+}, r_{0} ; e^{q^{\prime}}\right)>2 q$.

First consider the term $g_{2}$. Note that $g_{2}\left(\theta^{+}, r_{0}, 0\right)=0$. For $\theta\left(\theta^{+} ; t\right)=\theta^{+}+$ $t \xi\left(\theta^{+} ; t\right) \omega$ we have

$$
\kappa\left(q \theta_{1}\left(\theta^{+} ; t\right)-p \theta_{3}\left(\theta^{+} ; t\right)\right)=\left(-1 / 4+\kappa t \xi\left(\theta^{+} ; t\right)(q \alpha-p)\right) \bmod 1 .
$$


Then for $t=e^{q^{\prime}}$ we have

$$
\begin{aligned}
g_{2}\left(\theta^{+}, r_{0} ; t\right) & =\frac{q \kappa e^{-q^{\prime}}}{q \alpha-p} \cos 2 \pi \kappa\left(q \theta_{1}\left(\theta^{+} ; t\right)-p \theta_{3}\left(\theta^{+} ; t\right)\right) \\
& =\frac{q \kappa e^{-q^{\prime}}}{q \alpha-p} \sin \left(2 \pi \kappa t \xi\left(\theta^{+}, t\right)(q \alpha-p)\right) \\
& \geq \frac{q \kappa e^{-q^{\prime}}}{q \alpha-p} \pi \kappa t \xi\left(\theta^{+}, t\right)(q \alpha-p) \geq q \kappa^{2} e^{-q^{\prime}} t=q^{5}
\end{aligned}
$$

We used the evident estimate $\sin (x) \geq x / 2$ for $x \in[0, \pi / 2]$, and

$$
2 \pi \kappa t \xi\left(\theta^{+}, t\right)(q \alpha-p) \leq 4 \pi \kappa e^{q^{\prime}} e^{-2 q^{\prime}}=4 \pi q^{2} e^{-q^{\prime}} \in[0, \pi / 2] .
$$

To estimate the other terms in $r_{1}\left(\theta^{+}, r_{0} ; t\right)$, note that $\left|c_{1}+g_{1}\left(\theta^{+}, r_{0} ; 0\right)\right|=\left|r_{0,1}\right| \leq$ $q / 10$. By (4.3), the derivative $\dot{g}_{1}\left(\theta^{+}, r_{0}, t\right)$ satisfies $\left|\dot{g}_{1}\left(\theta^{+}, r_{0}, t\right)\right| \leq 2 \pi C q^{2} e^{-q^{\prime}} \leq$ $2 \pi q^{3} e^{-q^{\prime}}$. For $t=e^{q^{\prime}}$ we have $\Delta g_{1}\left(\theta^{+}, r_{0}, t\right):=\left|g_{1}\left(\theta^{+}, r_{0}, t\right)-g_{1}\left(\theta^{+}, r_{0}, 0\right)\right| \leq$ $2 \pi t q^{2} e^{-q^{\prime}}=2 \pi q^{2}$ and, finally,

$$
\begin{aligned}
r_{1}\left(\theta^{+}, r_{0} ; t\right)= & c_{1}+g_{1}\left(\theta^{+}, r_{0}, t\right)+g_{2}\left(\theta^{+}, r_{0}, t\right) \\
= & r_{0,1}+\Delta g_{1}\left(\theta^{+}, r_{0}, t\right)+g_{2}\left(\theta^{+}, r_{0}, t\right) \\
& \geq g_{2}\left(\theta^{+}, r_{0}, t\right)-\left|r_{0,1}\right|-\left|\Delta g_{1}\left(\theta^{+}, r_{0}, t\right)\right| \geq q^{5}-q / 10-2 \pi q^{3}>2 q .
\end{aligned}
$$

In the same way, there is a point $\left(\theta^{-}, r_{0}\right) \in \tilde{J}^{(1)}$ such that the solution $r_{1}\left(\theta^{-}, r_{0} ; t\right)$ with the initial condition $r_{1}\left(\theta^{-}, r_{0} ; 0\right)=r_{0,1}$ satisfies $r_{1}\left(\theta^{-}, r_{0} ; e^{q^{\prime}}\right) \leq-2 q$. This implies that $\Phi_{S}^{t}$ is $(1,1 / \kappa q, 2 q)$ stretching. In the same way, one verifies that $\Phi_{S}^{t}$ is $\left(2,1 / q^{\prime}, 2 q^{\prime}\right)$ stretching.

Proof of Proposition 3.6. The proof of Proposition 3.6 follows from Lemma 4.3 exactly as Proposition 3.3 followed from Lemma 4.1.

4.2. Stretching in the angle directions. In this section, we prove Proposition 3.7. Namely, we study the behavior of $\pi_{\theta}\left(\Phi_{\tilde{H}}^{t}\right)$, which is the solution of the equation

$$
\dot{\theta}=\frac{1}{\phi(\theta)} \omega \text {. }
$$

As this flow does not depend on $r$, we fix an arbitrary $r_{0}$ and omit it from the notations. To shorten the notation from $\S 2.3$, we denote

$$
\begin{gathered}
\Phi_{\phi}^{t}\left(\theta_{0}\right):=\pi_{\theta}\left(\Phi_{H}^{t}\left(r_{0}, \theta_{0}\right)\right), \\
B_{\theta, n}\left(\theta_{0}\right):=\pi_{\theta}\left(B_{n}\left(r_{0}, \theta_{0}\right)\right), \\
R_{\theta}\left(\theta_{0}, l, l^{\prime}\right):=\pi_{\theta}\left(R\left(r_{0}, \theta_{0}, l, l^{\prime}\right)\right) .
\end{gathered}
$$

Note that $R_{\theta}\left(\theta_{0}, l, l^{\prime}\right)$ is indeed a flat rectangle.

Proof of Proposition 3.7. As $\tilde{h}(\theta)$ does not depend on the $r$ variables, the restriction of the flow of $\tilde{H}=(1 / \phi(\theta))(\langle r, \omega\rangle+\tilde{h}(\theta))$ onto $\mathbb{T}^{3}$ is the same as that of $\bar{H}=(1 / \phi(\theta))\langle r, \omega\rangle$. We study the latter. 
First observe that, considering the global section $\left\{\theta_{3}=0\right\}$, one can see the flow of $\bar{H}$ to be equivalent to a special flow $T_{\left(\alpha, \alpha^{\prime}\right), \varphi}^{t}$ above the translation $T_{\left(\alpha, \alpha^{\prime}\right)}$ on $\mathbb{T}^{2}$ and under a ceiling function of the form

$$
\varphi(\theta)=1+\sum_{n=1}^{\infty} q_{n} e^{-q_{n}^{\prime}} \cos 2 \pi\left(q_{n} \theta_{1}\right) .
$$

The phase space $M_{\left(\alpha, \alpha^{\prime}\right), \varphi}$ of $T_{\left(\alpha, \alpha^{\prime}\right), \varphi}^{t}$ is $\mathbb{T}^{2} \times \mathbb{R}$ with the identification $\left(\theta_{1}, \theta_{2}, s+\right.$ $\left.\varphi\left(\theta_{1}, \theta_{2}\right)\right) \sim\left(\theta_{1}+\alpha, \theta_{2}+\alpha^{\prime}, s\right)$. These flows were studied in [F] and the proof of the proposition follows from [F]. For completeness, we sketch the proof here. Observe that, as proved in Proposition 3.7 of [F], for intervals $I_{n} \subset \mathbb{R}$ of length $(1 / 2-2 / n) q_{n}^{-1}$ of the form $\left|\left\|q_{n} \theta_{1}\right\|\right| \in\left[1 / n, \frac{1}{2}-(1 / n)\right]$ or $\left\|q_{n} \theta_{1}\right\| \mid \in\left[\frac{1}{2}+(1 / n), 1-(1 / n)\right]$, and for $m \in\left[t_{n} / 2,2 t_{n}\right]$, it holds for some constant $C>0$ that for every $\theta_{1} \in I_{n}$,

$$
C^{-1} \frac{q_{n}^{2}}{n} \leq\left|\partial_{\theta_{1}} \varphi_{m}(\theta)\right| \leq C q_{n}^{2},
$$

where $\varphi_{m}$ denotes the $m$ th Birkhoff sum of the function $\varphi$. The latter estimate follows from the very good rational approximation of $\omega_{1}$, because (2.1) implies that $\left\|q_{n} \omega_{1}\right\| \leq$ $e^{-q_{n}^{\prime}} / 4$. Now, the left-hand side of (4.6) implies that $T_{\left(\alpha, \alpha^{\prime}\right), \varphi}^{t_{n}}\left(I_{n} \times\left\{\theta_{2}\right\} \times\{s\}\right)$, for any $\theta_{2} \in \mathbb{T}$ and any $s \leq C$, is a union of more than $\sqrt{q_{n}}$ almost vertical strips that follow the orbit of $I_{n}$ under the base translation $T_{\left(\alpha, \alpha^{\prime}\right)}$. As $\sqrt{q_{n}} \gg \exp \circ \exp (n)$, we get that $T_{\left(\alpha, \alpha^{\prime}\right), \varphi}^{t_{n}}\left(I_{n} \times\left\{\theta_{2}\right\} \times\{s\}\right)$ is $e^{-2 n}$ dense in the space $M_{\left(\alpha, \alpha^{\prime}\right), \varphi}$. This fact, plus the right-hand side of (4.6), together with the fact that there is no shear in the $\theta_{2}$ direction (the ceiling function depends only on $\theta_{1}$ ), imply that for any box $B_{\theta, n}$, and for any $s \in \mathbb{R}$, there exists a rectangle $R_{n}^{\prime}$ of size $1 / q_{n}^{3} \times 1 / q_{n}^{3}$ such that

$$
T_{\left(\alpha, \alpha^{\prime}\right), \varphi}^{t_{n}}\left(R_{n}^{\prime} \times\{s\}\right) \subset B_{\theta, n} .
$$

Going back to the original flow on $\mathbb{T}^{d}$, this implies the requirement of the proposition.

Acknowledgements. This work as supported in part by the Knut and Alice Wallenberg foundation, grant KAW 2016.0403, and by the ANR, grant ANR-15-CE40-0001. This work was also supported in part by the Swedish Research Council, grant VR 2015-04012.

\section{REFERENCES}

[A] V. I. Arnold. Mathematical problems in classical physics. Trends and Perspectives in Applied Mathematics (Applied Mathematical Sciences, 100). Springer, New York, 1994, pp. 1-20.

[AK] D. V. Anosov and A. B. Katok. New examples in smooth ergodic theory. Ergodic diffeomorphisms. Trans. Moscow Math. Soc. 23 (1970), 1-35.

[BKZ] P. Bernard, V. Kaloshin and K. Zhang. Arnold diffusion in arbitrary degrees of freedom and normally hyperbolic invariant cylinders. Acta Math. 217(1) (2016), 1-79.

[EFK] H. Eliasson, B. Fayad and R. Krikorian. On the stability of KAM tori. Duke Math. J. 164(9) (2015), 1733-1775.

[F] B. Fayad. Analytic mixing reparametrizations of irrational flows ETDS. Ergod. Th. \& Dynam. Sys. 22(2) (2002), 437-468.

[FF] G. Farré and B. Fayad. Instabilities for analytic quasi-periodic invariant tori. Preprint, 2019, arXiv:1912.01575. J. Eur. Math. Soc., to appear. 
[FS] B. Fayad and M. Saprykina. Isolated elliptic fixed points for smooth Hamiltonians. Modern Theory of Dynamical Systems (Contemporary Mathematics, 692). American Mathematical Society, Providence, RI, 2017, pp. 67-82.

[H] M. Herman. Some Open Problems in Dynamical Systems (Proc. Int. Congress of Mathematicians, Vol. II, Berlin, 1998). Doc. Math. Extra Vol II (1998), 797-808.

[Ha] P. Halmos. Lectures on Ergodic Theory. Chelsea Publishing, New York, 1956.

[K1] A. B. Katok. Spectral properties of dynamical systems with an integral invariant on the torus. Funct. Anal. Appl. 1 (1967), 296-305.

[K2] A. B. Katok. Ergodic perturbations of degenerate integrable Hamiltonian systems. Math. USSR Izv. 7(3) (1973), 535-571.

[Koc] A. V. Kochergin. On the absence of mixing in special flows over the rotation of a circle and in flows on a two-dimensional torus. Dokl. Akad. Nauk 205(3) (1972), 515-518.

[KZZ] V. Kaloshin, K. Zhang and Y. Zheng. Almost dense orbit on energy surface. Proc. XVIth Int. Congress on Mathematical Physics (Prague, Czech Republic, 3-8 August 2009). Ed. P. Exner. World Scientific Publishing Co., Hackensack, NJ, 2010, pp. 314-322.

[Y] J.-C. Yoccoz. Petits diviseurs en dimension 1 (Astérisque, 231). Société mathématique de France, Paris, 1995. 